\title{
Nonintrusive Installation of the TMR Busbar DC Current Sensor
}

\author{
X. P. Xu $\mathbb{D}^{1},{ }^{1}$ T. Z. Liu $\mathbb{D},{ }^{1}$ M. Zhu $\mathbb{D}^{2},{ }^{2}$ and J. G. Wang $\mathbb{D}^{2}$ \\ ${ }^{1}$ The School of Mechatronic Engineering and Automation, Shanghai University, Shanghai 200044, China \\ ${ }^{2}$ Sinomags Technology Co., Ltd., Ningbo 315200, China
}

Correspondence should be addressed to T. Z. Liu; liutz09@126.com

Received 16 September 2020; Revised 20 December 2020; Accepted 25 January 2021; Published 8 February 2021

Academic Editor: Heinz C. Neitzert

Copyright ( $\odot 2021$ X. P. Xu et al. This is an open access article distributed under the Creative Commons Attribution License, which permits unrestricted use, distribution, and reproduction in any medium, provided the original work is properly cited.

\begin{abstract}
In recent years, new energy vehicles, photovoltaic power stations, communication base stations, energy storage systems, and other power electronic systems have developed rapidly. The development of these systems has the trend of continuously increasing the power density per unit area, reducing the system volume, and continuously increasing using the busbars. More and more new current sensors will be used in these systems and play a key role. Traditional current sensor cannot meet the development trend of power electronic systems due to their large size and high cost. In this paper, a new small coreless tunnel junction magnetoresistance (TMR) busbar dc current sensor adopted differential scheme which improves the sensor's anti-interference ability that is designed. The current sensor adopts an open-closed structure for easy nonintrusive installation. Four TMRs which adopted differential structure are placed on the edges of the busbar. The peak current measurement range is $\pm 600 \mathrm{~A}$, the rated current measurement range is $\pm 300 \mathrm{~A}$, and the supply voltage is $5 \mathrm{~V}$.
\end{abstract}

\section{Introduction}

Due to its noncontact isolation measurement, high precision, and low cost $[1,2]$, current sensors are widely used in power electronic systems, such as photovoltaics, wind power, and electric vehicles, and the use of current sensors has increased year by year. A magnetic field is generated by the measured current. The magnetic field can be measured using different sensor technologies including Hall effect sensor, magnetic fluxgate, and magnetoresistance (MR) sensor. The MR sensors have grabbed the attention of researchers in recent years due to their high sensitivity. The latest research in this field mainly includes anisotropic magnetoresistance (AMR), giant magnetoresistance (GMR), and TMR. Hall, AMR, GMR, and TMR have been used in current sensors [3-6]. Hall has the inherent defects of low sensitivity, high power consumption, and poor linearity. Although the sensitivity of AMR and GMR is much higher than that of hall, their linear range is narrow. Compared with hall, AMR, and GMR, TMR has better temperature stability, higher sensitivity, lower power consumption, and better linearity. The conventional busbar current sensor with differential structure adopts a pair of hall sensors on the conductor surface, which partially suppresses the external magnetic field [7-9]. A busbar with an amphitheater hole is shown [10], and an AMR busbar current sensor is described [6]. Circular sensor array is also a commonly used measurement method. Its accuracy is less dependent on the position of the conductor, and it has better resistance to external magnetic field interference $[11,12]$. The disadvantage of circular arrays is that they are too large. In previous research literature, each magnetic sensing device is separately amplified and measured, and then the measured current value is obtained by comprehensive calculation and fitting [13]. The existing method is complicated, and the cost is high. Since the TMR itself is a magnetoresistance device, the magnetoresistances can be connected in parallel. In this paper, four TMRs are directly connected in parallel.

The TMR sensor is a thin film element in which a barrier layer consisting of a thin insulator is sandwiched between two ferromagnetic layers (free layer/pin layer). Due to the wave-like nature of electrons, electrons in the ferromagnetic layer can tunnel through the insulator layer, and the possibility of tunneling through is related to the magnetization of pin and the free layer. The magnetization direction of the pin layer is fixed, and the magnetization direction of free layer changes with the direction of the external magnetic field. 


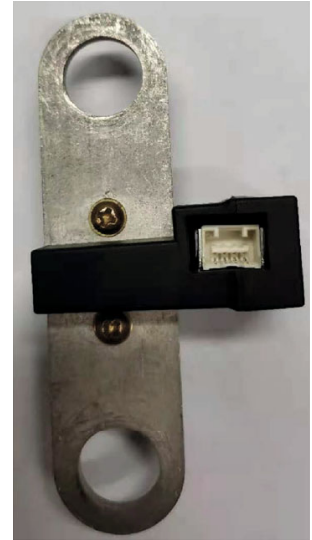

(a)

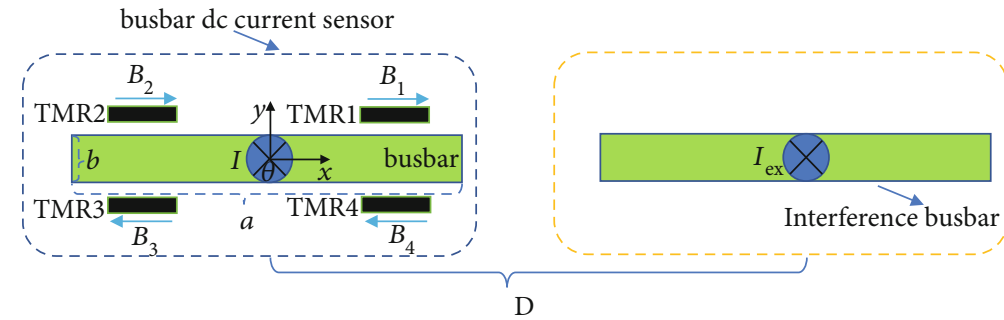

(b)

Figure 1: (a) Current sensor photo. (b) Structure of the busbar current sensor with the interference busbar.

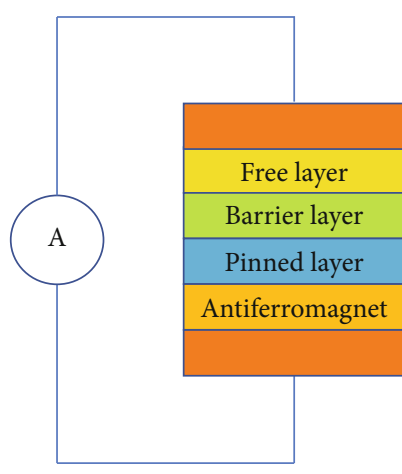

(a)

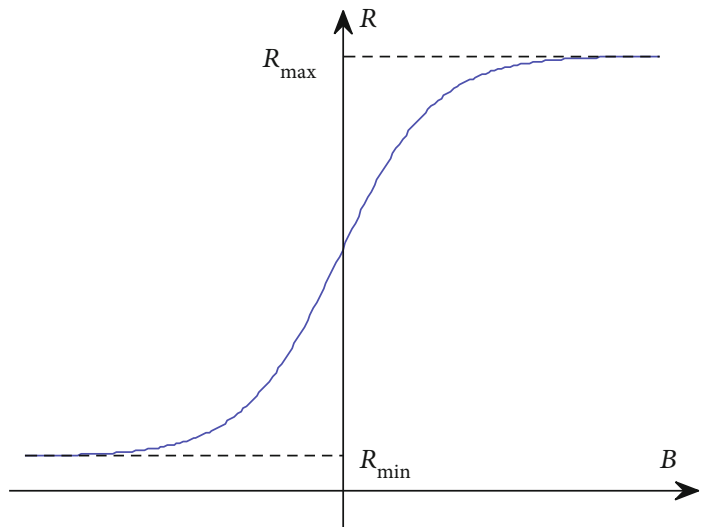

(b)

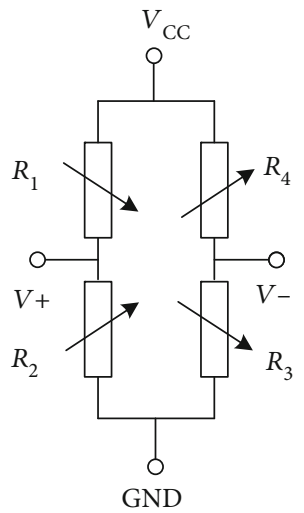

(c)

FIgure 2: (a) Structure of an MTJ. (b) Typical response curve of a TMR sensor. (c) Wheatstone bridge circuit of TMR.

When the direction of magnetization of the pin layer is parallel to that of the free layer, the resistance is minimal, since the electron tunneling is the largest and vice versa. Obviously, the TMR element has a sensitive orientation due to pin layer magnetization fixation. In this paper, the sensitive direction of all TMR chips is parallel to the surface and set to the $x$ direction (Figure 1). The structure of the TMR sensor is a magnetic multilayer film material, as shown in Figure 2(a). There is a thin layer of insulation that can be tunneled by electrons between the magnetic layers. This structure is called a magnetic tunnel junction (MTJ). When the free layer is magnetically aligned with the pinned layer, electrons are more likely to pass through the barrier layer $[14,15]$.

Therefore, the resistance $(R)$ of the sensor depends on the external magnetic flux field $(B)$, as shown in Figure 2(b). In 


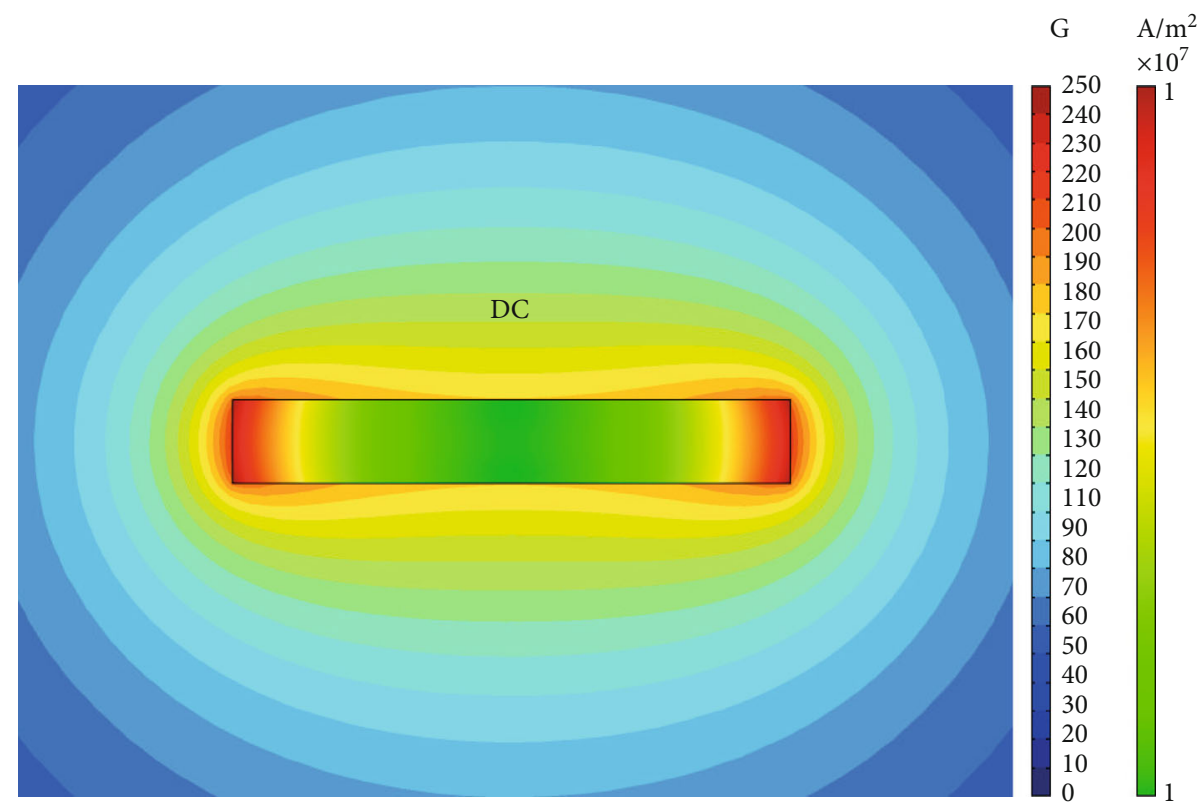

FIgURe 3: 600 A current density at busbar and magnetic flux density near busbar.

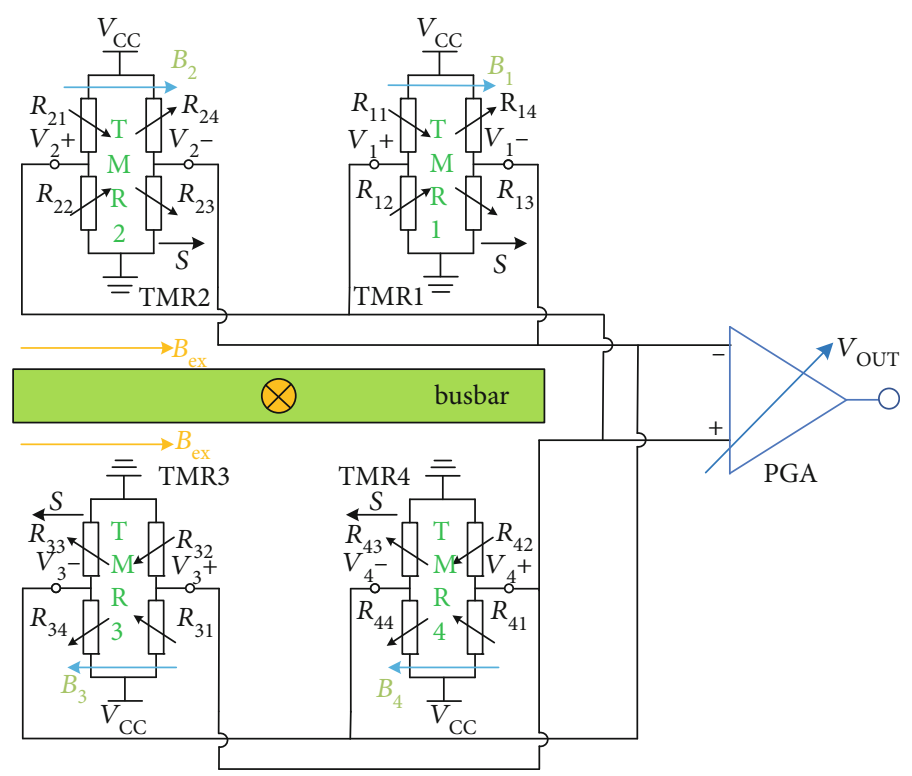

FIgURE 4: Circuit diagram of current sensor module.

Figure 2(c), the TMR chip consists of Wheatstone bridge and has a good temperature characteristics and anticommonmode signal interference. The bridge circuit contains four resistances as shown. $\mathrm{V}+$ represents the forward half-bridge terminal output of TMR, and V- represents the negative halfbridge terminal output of TMR. The voltage between $\mathrm{V}+$ and $\mathrm{V}$ - is proportional to the adjacent magnetic flux density, also to the current under measurement.

The new type of current sensor proposed in this paper adopts the TMR array, with flexible differential structure and no magnetic core, and is suitable for current measure- ment in different ranges. The use of nonmagnetic core solution can effectively reduce the volume and weight. Differential structure current sensors are used to partially suppress the external magnetic field interference and improve the test accuracy. We present a new small volume current sensor with the characteristics of the nonintrusive installation. The supply voltage is $5 \mathrm{~V}$, the rated current measurement range is $\pm 300 \mathrm{~A}$, and the peak current measurement range is $\pm 600 \mathrm{~A}$, corresponding to $2.5 \pm 2 \mathrm{~V}$ output at $\pm 600 \mathrm{~A} \mathrm{dc}$ current. It uses a differential technique based on tunnel junction magnetoresistance (TMR) 


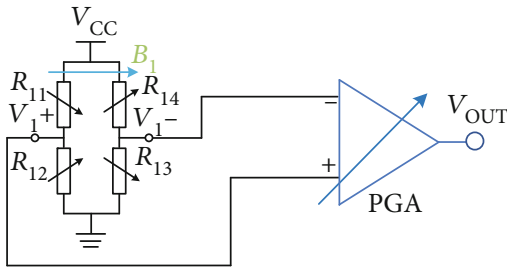

(a)

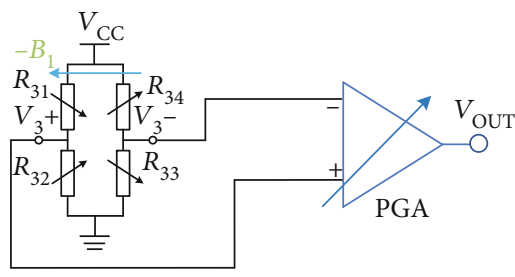

(c)

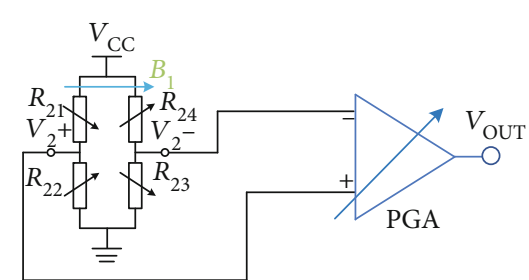

(b)

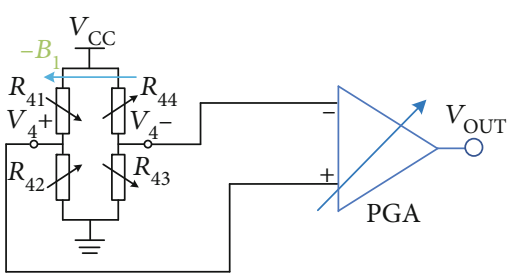

(d)

Figure 5: TMR and PGA connection.

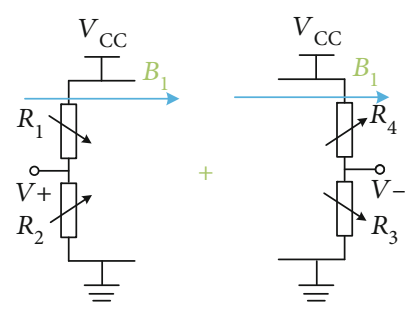

(a)
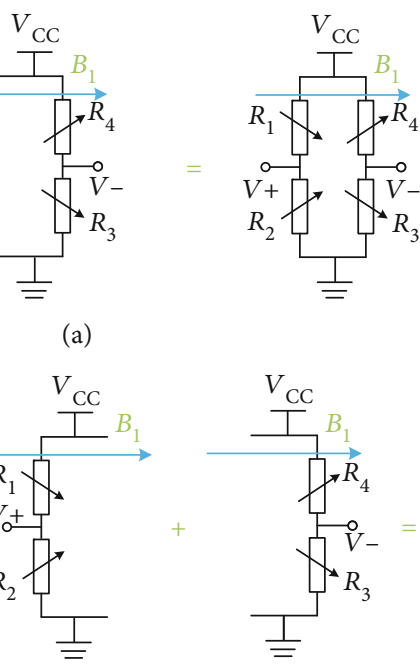

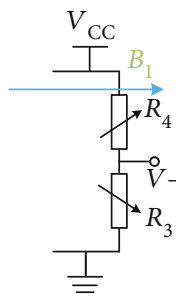

(c)

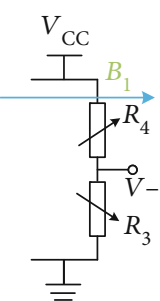

(b)

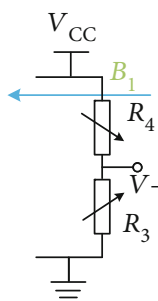

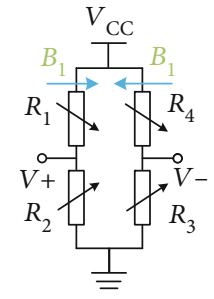

FIgUre 6: The equivalent circuit of TMR in the magnetic flux density.

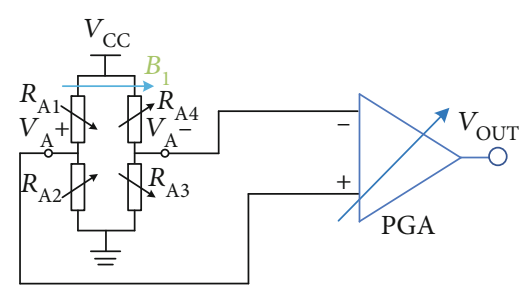

FIgURE 7: The equivalent circuit of the busbar current sensor.

technology placed on the surface of the busbar. The current sensor module is consisted of TMR chips, programmable gain amplifier (PGA). Because of busbar's skin effect, which is relatively complex, this paper focuses on $\mathrm{dc}$ current measurement, not AC current measurement.

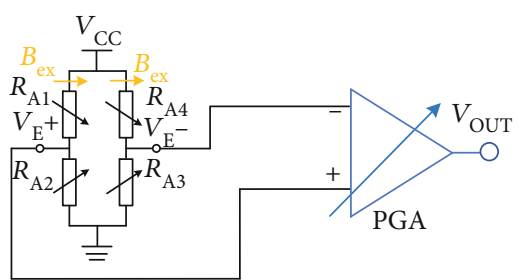

FIgURE 8: The equivalent circuit of the busbar current sensor in the external magnetic flux density.

\section{Sensor Design}

2.1. Busbar Current Sensor. The TMR and PGA chips are soldered on the printed circuit board (PCB). The four TMR chips are connected in differential mode and then connected to the PGA input port. PGA is used to adjust the gain and 
temperature characteristics of the current sensor. For the current sensor, TMR and PGA used in this paper manufactured by Sinomags Technology Co., Ltd. in China. We design and test the performance of TMR and PGA and give the test data of the current sensor in this paper.

Figure 1 shows the photo of the current sensor and the schematic diagram of the differential structure of the module. The $V+$ terminals of TMR1, TMR2, TMR3, and TMR4 are connected with the IN+ terminal of PGA. The $V-$ terminals of TMR1, TMR2, TMR3, and TMR4 are connected with the IN- terminal of PGA. This connection forms a differential structure. The use of a differential structure has the advantage of being resistant to external magnetic fields. $I$ is the measured busbar current, and the cross-sectional area $(a \times b)$ of the busbar is $20 \mathrm{~mm} \times 3 \mathrm{~mm}$, whose length is $80 \mathrm{~mm}$, and the vertical distance ( $y$-axis) between the TMR and the center of the busbar is $4.8 \mathrm{~mm}$, which is determined by the thickness of the parts. The size of the busbar is 20 $\mathrm{mm} \times 3 \mathrm{~mm} \times 80 \mathrm{~mm}$, and the TMR sensitivity direction is parallel to the $x$-axis in Figure $1 .(0,0)$ is the busbar center coordinate. The coordinates of four TMR chips are (6.3, $4.8),(-6.3,4.8),(-6.3,-4.8)$, and $(6.3,-4.8) . I_{\mathrm{ex}}$ is the interference busbar current. The distance $(D)$ between the centers of two busbars with the same size is $60 \mathrm{~mm}$, which is three times the busbar width.

The sensitivity of TMR is typically $0.1 \mathrm{~m} V / V / \mathrm{Gs}$, and the saturation magnetic field is $200 \mathrm{Gs}$. In the $200 \mathrm{Gs}$ magnetic field range, the TMR chip has good linearity, beyond $200 \mathrm{Gs}$, and the TMR chip output tends to be saturated. When the measured current is $600 \mathrm{~A}$, the working magnetic field of the sensor is within 200 Gs (Figure 3); so, the current sensor has good linearity. Four TMR chips are connected in a differential mode and mounted on the sides of the PCB. The current sensor adopts an open-closed structure for easy installation. The sensor is installed at busbar via 2 set screws.

2.2. Magnetic-Field Analysis. For the busbar whose crosssection is rectangular, $a$ and $b$ are the length and width dimensions of the cross-section of the busbar. Current $I$ is passed through the busbar. $B_{x}$ represents the magnetic flux density parallel to the TMR sensitivity at coordinates $(f x, f y)$ in Figure 1. According to Maxwell equation, the magnetostatic equation can be written as equation (1).

$B_{x}=\int_{-a / 2}^{a / 2} \int_{-b / 2}^{b / 2} \frac{2 I}{\sqrt{(f x-x)^{2}+(f y-y)^{2}}} \cdot \frac{f y-y}{\sqrt{(f x-x)^{2}+(f y-y)^{2}}} d x d y$.

COMSOL software was used for finite element simulation. Figure 3 shows $600 \mathrm{~A}$ current densities at the busbar and the magnetic flux density near busbar. Take the magnetic flux density of $B_{1}$ and $B_{2}$ in the positive direction. Referring to equation (1), when current $I$ is $600 \mathrm{~A}$, the absolute magnetic flux density is show in Figure 3.

2.3. Sensor Circuit Design. The equivalent circuit diagram of the current sensor is shown in Figure 4. The TMR chip con- sists of four resistors connected as a Wheatstone bridge. Each TMR chip is connected to PGA through the $V+$ terminal and $V$ - terminal. Ideally, the sensitivity of TMR1, TMR2, TMR3, and TMR4 is the same and parallel to the $x$-axis. The $V+$ terminals of TMR1, TMR2, TMR3, and TMR4 are connected to the IN+ terminal of the PGA. The V-terminals of TMR1, TMR2, TMR3, and TMR4 are connected to the INterminal of the PGA. $B_{1}, B_{2}, B_{3}$, and $B_{4}$ represent the magnetic flux density at the positions of TMR1, TMR2, TMR3, and TRM4, respectively. $B_{2}$ and $B_{1}$ have the same value, in the same direction. $B_{3}$ and $B_{1}$ are equal and opposite. $B_{4}$ and $B_{1}$ are equal and opposite. $B_{\text {ex }}$ represents the external magnetic flux intensity. $S$ represents the sensitivity of the TMR chip.

$$
B_{1}=B_{2}=B_{3}=B_{4}
$$

The equivalent circuit of each TMR and PGA connection is shown in Figure 5.

Ideally, the resistance values of the four resistors of TMR chips in the same wafer are equal. The same goes for the sensitivity.

$$
\begin{aligned}
R_{11} & =R_{12}=R_{13}=R_{14}=R_{21}=R_{22}=R_{23}=R_{24} \\
& =R_{31}=R_{32}=R_{33}=R_{34}=R_{41}=R_{42}=R_{43}=R_{44}
\end{aligned}
$$

Each $V+$ and $V-$ output for each TMR chip is shown in equations (4) to (12).

$$
\begin{gathered}
V_{1}+=\frac{V_{\mathrm{CC}}}{2}+V_{\mathrm{CC}} B_{1} \frac{S}{2}, \\
V_{1}-=\frac{V_{\mathrm{CC}}}{2}-V_{\mathrm{CC}} B_{1} \frac{S}{2}, \\
V_{1}+-V_{1}-V_{\mathrm{CC}} B_{1} S, \\
V_{2}+=\frac{V_{\mathrm{CC}}}{2}+V_{\mathrm{CC}} B_{2} \frac{S}{2}=\frac{V_{\mathrm{CC}}}{2}+V_{\mathrm{CC}} B_{1} \frac{S}{2}, \\
V_{2}-=\frac{V_{\mathrm{CC}}}{2}-V_{\mathrm{CC}} B_{2} \frac{S}{2}=\frac{V_{\mathrm{CC}}}{2}-V_{\mathrm{CC}} B_{1} \frac{S}{2}, \\
V_{3}+=\frac{V_{\mathrm{CC}}}{2}+V_{\mathrm{CC}} B_{3} \frac{S}{2}=\frac{V_{\mathrm{CC}}}{2}+V_{\mathrm{CC}} B_{1} \frac{S}{2}, \\
V_{3}=\frac{V_{\mathrm{CC}}}{2}-V_{\mathrm{CC}} B_{3} \frac{S}{2}=\frac{V_{\mathrm{CC}}}{2}-V_{\mathrm{CC}} B_{1} \frac{S}{2}, \\
V_{4}+=\frac{V_{\mathrm{CC}}}{2}+V_{\mathrm{CC}} B_{4} \frac{S}{2}=\frac{V_{\mathrm{CC}}}{2}+V_{\mathrm{CC}} B_{1} \frac{S}{2}, \\
V_{4}-=\frac{V_{\mathrm{CC}}}{2}-V_{\mathrm{CC}} B_{4} \frac{S}{2}=\frac{V_{\mathrm{CC}}}{2}-V_{\mathrm{CC}} B_{1} \frac{S}{2} .
\end{gathered}
$$

$V_{1}+, V_{2}+, V_{3}+$, and $V_{4}+$ are connected in parallel and then connected to the IN+ of the PGA. $V_{1}-, V_{2}-$, $V_{3}-$, and $V_{4}-$ are connected in parallel and then connected to the IN- of the PGA.

The equivalent circuit of TMR in the magnetic flux density is shown in Figure 6. 


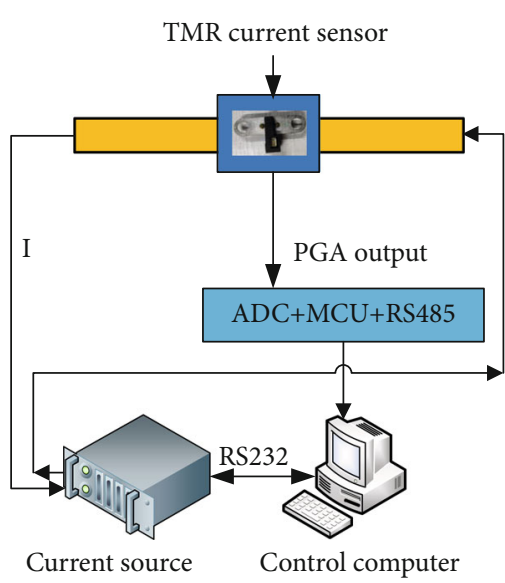

(a)

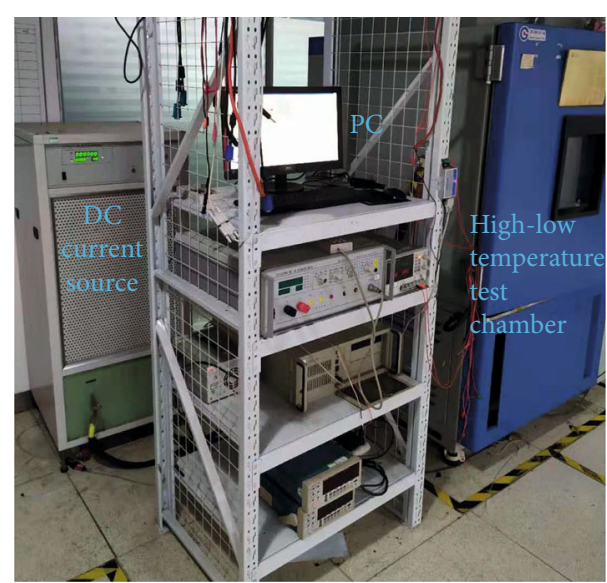

(b)

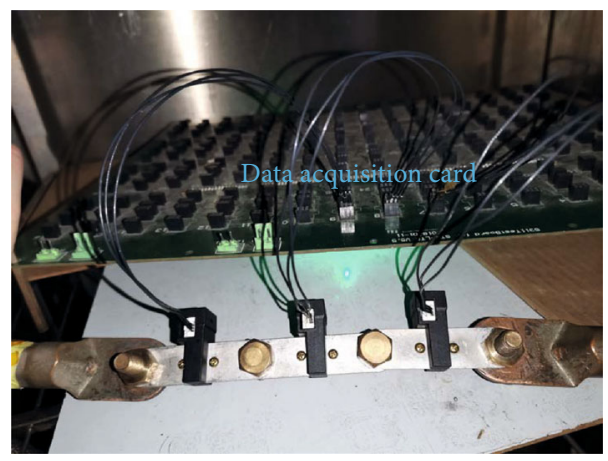

(c)

Figure 9: (a) Diagram of the measurement system. (b) Test device. (c) Busbar current sensor test.

TABLE 1: Test instruments.

\begin{tabular}{lcc}
\hline Device & Model & Manufacturer \\
\hline DC current source & XF30ZA-1 K & Tsinghua Science and Technology Development Co., Ltd. \\
Power supply & GPD-3303S & Guwei Electronics Co., Ltd. \\
Multimeter & DMM4050 & Tektronix \\
PC & RK-610 & ADLINK \\
Data acquisition card & SMDAQ-6000 & Sinomags Technology Co., Ltd. \\
High-low temperature & RGDJ-500 & Surui Test Equipment Co., Ltd. \\
\hline
\end{tabular}

The equivalent circuit of the busbar current sensor is shown in Figure 7.

$$
\begin{gathered}
R_{A 1}=R_{11} / / R_{21} / / R_{31} / / R_{41}, \\
R_{A 2}=R_{12} / / R_{22} / / R_{32} / / R_{42}, \\
R_{A 3}=R_{13} / / R_{23} / / R_{33} / / R_{43}, \\
R_{A 4}=R_{14} / / R_{24} / / R_{34} / / R_{44}, \\
V_{A^{+}}=V_{1}+/ / V_{2}+/ / V_{3}+/ / V_{4}+, \\
V_{A}-=V_{1}-/ / V_{2}-/ / V_{3}-/ / V_{4}-, \\
B_{1}=I \cdot K_{I} .
\end{gathered}
$$

$B_{1}$ represents the magnetic flux density generated by the measured current $I$ at TMR1 according to equation (1). $K_{I}$ is the coefficient between $B_{1}$ and current $I$.

$$
V_{\text {OUT }}=\left(V_{A}+-V_{A}-\right) K_{A} \text {. }
$$

$K_{A}$ is the magnification of PGA.

Equations (4)-(6) and (17)-(20) can be calculated to obtain equation (21). The $V_{\text {OUT }}$ of the busbar current sensor is shown in equation (21).

$$
V_{\text {OUT }}=\left(V_{1}+-V_{1}-\right) K_{A}=V_{C C} B_{1} S K_{A}=V_{C C} \mathrm{ISK}_{I} K_{A} .
$$




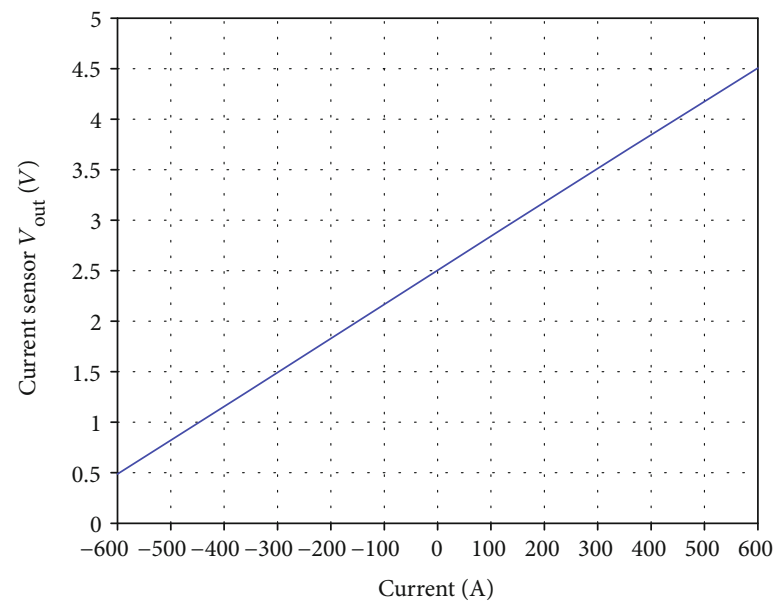

(a)

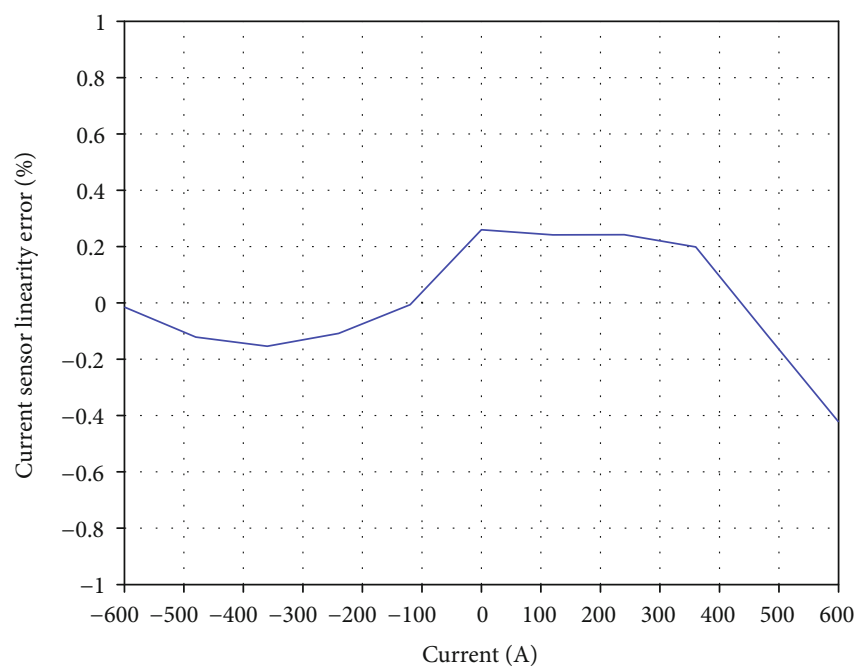

(b)

FIgURE 10: (a) $600 \mathrm{~A}$ dc peak current $I$ - $V$ characteristics of the current sensor. (b) Relative accuracy of current sensor within $600 \mathrm{~A}$ dc peak current at $25^{\circ} \mathrm{C}$.

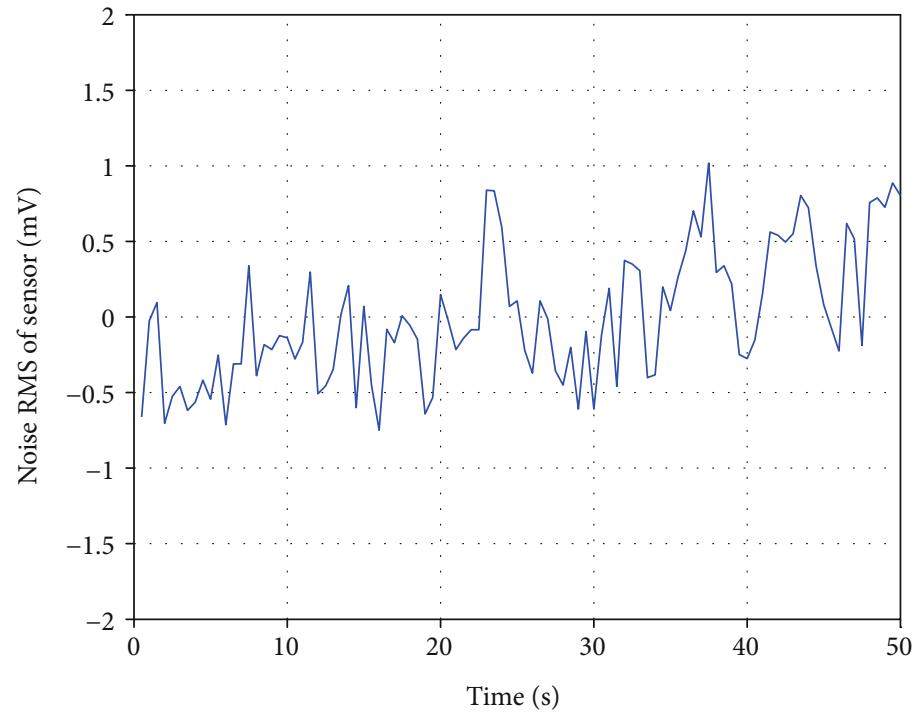

Figure 11: Noise RMS of the current sensor. 


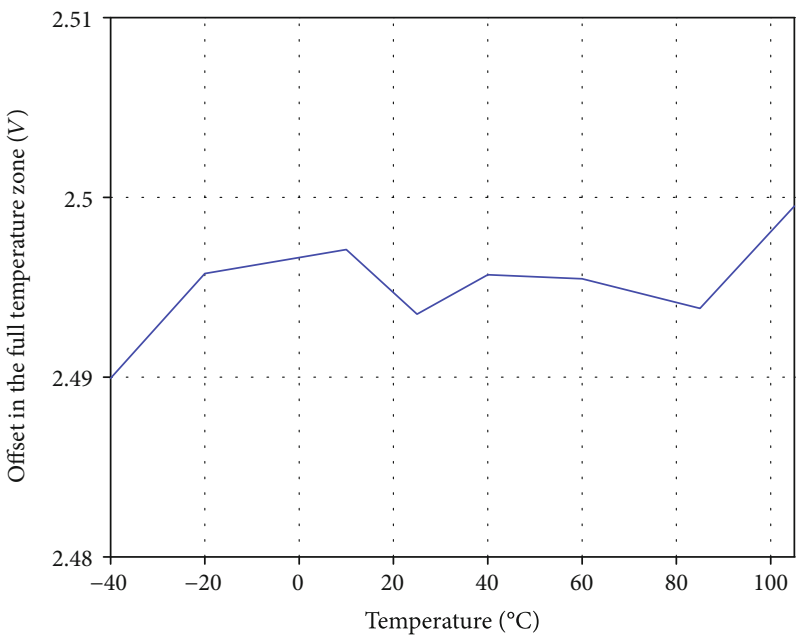

(a)

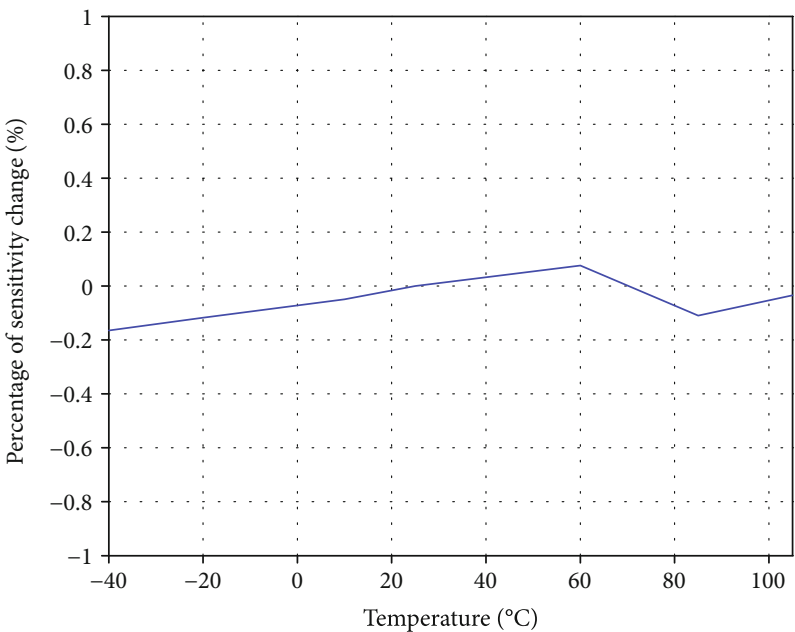

(b)

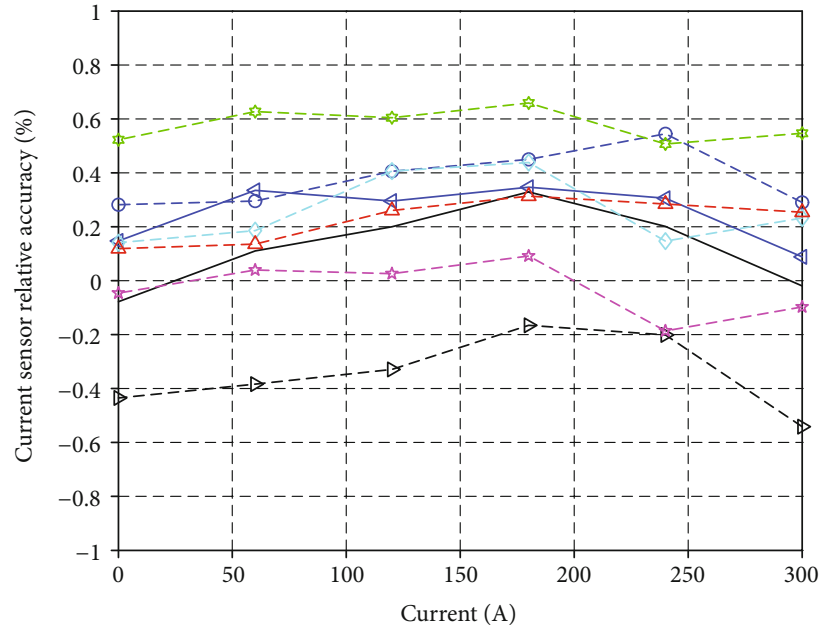

$$
\begin{array}{ll}
--40^{\circ} \mathrm{C} & -40^{\circ} \mathrm{C} \\
--20^{\circ} \mathrm{C} & -60^{\circ} \mathrm{C} \\
-\theta-10^{\circ} \mathrm{C} & --85^{\circ} \mathrm{C} \\
-25^{\circ} \mathrm{C} & --105^{\circ} \mathrm{C}
\end{array}
$$

(c)

FIguRe 12: (a) Offset in the full temperature zone. (b) Percentage of sensitivity change in the full temperature zone relative to $25^{\circ} \mathrm{C}$. (c) Relative accuracy of current sensor in the full temperature zone relative to $25^{\circ} \mathrm{C}$. 
The equivalent circuit of the busbar current sensor in the external magnetic flux density is shown in Figure 8.

$$
\begin{gathered}
V_{E}+=\frac{V_{\mathrm{CC}}}{2}+V_{\mathrm{CC}} B_{\mathrm{ex}} \frac{S}{2}, \\
V_{E}-=\frac{V_{\mathrm{CC}}}{2}+V_{\mathrm{CC}} B_{\mathrm{ex}} \frac{S}{2}, \\
V_{\text {OUT }}=\left(V_{E}+-V_{E}-\right) K_{A}=0 .
\end{gathered}
$$

Therefore, it can be concluded from equation (24) that under the constant external magnetic field interference, the output of the current sensor does not change, which meets the requirement of the resisting external constant magnetic field interference.

In Figure 1, when the measured busbar is passed with $600 \mathrm{~A}$ current without an external interference busbar, it can be obtained from Figure 3 that the equivalent differential magnetic field strength of the current sensor is $19.52 \mathrm{Gs}$. When the measured busbar and the interference busbar with the same size are passed with $600 \mathrm{~A}$ current in the same direction, the equivalent differential magnetic field strength of the current sensor is $19.80 \mathrm{Gs}$. The interference error of the current sensor is $1.4 \%$. When $D$ takes other values, the same method can be used for analysis and calculation.

\section{Results}

The maximum magnetic flux density generated by the $\mathrm{dc}$ 600 A current near TMR is less than $200 \mathrm{Gs}$, in the good working linear range of TMR.

Figure 9 shows the measurement system which includes current source, power supply, multimeter, PC, and data acquisition card which includes microcontroller unit (MCU), analog-to-digital converter (ADC), RS232, and RS485. The MCU is S9S08DZ60F2MLH made by NXP semiconductors, and the ADC is AD7194BCPZ made by Analog Devices, Inc. The dc current source is XF30ZA-1 K, which is manufactured by Tsinghua Science and Technology Development Co., Ltd., China, with a typical accuracy of $\pm 0.02 \%$, which can meet the requirements of current sensor testing in Table 1.

Figure 10 shows the $I-V$ characteristic, and the linearity error of the current sensor is less than $\pm 1 \%$ at $25^{\circ} \mathrm{C}$.

Using the default configuration of the multimeter DMM4050, a sampling period of $0.5 \mathrm{~s}$, sampling for $50 \mathrm{~s}$, to test the noise of the current sensor. Figure 11 shows the noise RMS (root mean square) of the current sensor that is about $\pm 1 \mathrm{mV}$. The good noise characteristic of the sensor is the guarantee of the good accuracy of the sensor.

Figure 12(a) shows the offset of the current sensor in the full temperature zone. The change of offset in the full temperature zone relative to $25^{\circ} \mathrm{C}$ is not more than $\pm 10 \mathrm{mV}$. The sensitivity change in the full temperature zone relative to $25^{\circ} \mathrm{C}$ is less than $\pm 1 \%$ in Figure 12 (b). Figure 12 (c) shows the relative accuracy of current sensor relative to $25^{\circ} \mathrm{C}$ in the full temperature zone. The relative accuracy of the current sensor in the full temperature range is within $\pm 1 \%$. So, the current sensor has good temperature characteristics.

\section{Conclusions}

This paper designs a new type of small volume dc current sensor, which has the characteristics of small volume, flexible installation, and high precision. The current sensor adopts a new differential combination scheme, which has good linearity and anti-interference ability. The current sensor adopts an open-closed structure for easy nonintrusive installation. Compared with the traditional magnetic current sensor and ring array sensor, the new sensor has a smaller volume. The size of the current sensor can be adjusted flexibly according to the size of the busbar. The relative accuracy of the current sensor in the full temperature range is within $\pm 1 \%$. It is very suitable for the dc busbar power system and has a broad application prospect.

\section{Data Availability}

The data that support the findings of this study are available from the corresponding author upon reasonable request.

\section{Conflicts of Interest}

The authors declare that they have no conflicts of interest.

\section{Acknowledgments}

The authors would like to thank H.Y. Chen, X.G. Yao, J.M. Bai, K. Tan, C. Gao, and J.J. Feng. This work went well under their help.

\section{References}

[1] C. Xiao, L. Zhao, T. Asada, W. G. Odendaal, and J. D. van Wyk, "An overview of integratable current sensor technologies," in 38th IAS Annual Meeting on Conference Record of the Industry Applications Conference, pp. 1251-1258, Salt Lake City, UT, USA, USA, 2003.

[2] S. Ziegler, R. C. Woodward, H. H. C. Iu, and L. J. Borle, "Current sensing techniques: a review," IEEE Sensors Journal, vol. 9, no. 4 , pp. 354-376, 2009.

[3] H. Zhang, F. Li, H. Guo, Z. Yang, and N. Yu, "Current measurement with 3-D coreless TMR sensor array for inclined conductor," IEEE Sensors Journal, vol. 19, no. 16, pp. 66846690, 2019.

[4] I. Jedlicska, R. Weiss, and R. Weigel, "Increasing the measurement accuracy of GMR current sensors through hysteresis modeling," in 2008 IEEE International Symposium on Industrial Electronics, pp. 884-889, Cambridge, UK, 2008.

[5] L. Cristaldi, A. Ferrero, M. Lazzaroni, and R. T. Ottoboni, “A linearization method for commercial hall-effect current transducers," IEEE Transactions on Instrumentation and Measurement, vol. 50, no. 5, pp. 1149-1153, 2001.

[6] Z. Zhenhong, O. Syuji, A. Osamu, and K. Hideto, "Development of the highly precise magnetic current sensor module of +/-300 A utilizing AMR element with bias-magnet," IEEE Transactions on Magnetics, vol. 51, no. 1, 2015.

[7] “CSA-1V Melexis Datasheet,” June 2013, https://www.melexis .com.

[8] M. Blagojevic, U. Jovanovic, I. Jovanovic, D. Mancic, and R. S. Popovic, "Coreless open-loop current transducers based on 
hall effect sensor CSA-1V," Facta Universitatis Series: Electronics and Energetics, vol. 29, no. 4, pp. 489-507, 2016.

[9] M. Blagojevic, U. Jovanovic, I. Jovanovic, D. Mancic, and R. S. Popovic, "Realization and optimization of bus bar current transducers based on Hall effect sensors," Measurement Science and Technology, vol. 27, no. 6, p. 065102, 2016.

[10] P. Ripka, M. Pribil, V. Petrucha, V. Grim, and K. Draxler, “A fluxgate current sensor with an amphitheater Busbar," IEEE Transactions on Magnetics, vol. 52, no. 7, pp. 53-58, 2016.

[11] L. Di Rienzo and Z. Zhang, "Spatial harmonic expansion for use with magnetic sensor arrays," IEEE Transactions on Magnetics, vol. 46, no. 1, pp. 53-58, 2010.

[12] R. Weiss, R. Makuch, A. Itzke, and R. Weigel, "Crosstalk in circular arrays of magnetic sensors for current measurement," IEEE Transactions on Industrial Electronics, vol. 64, no. 6, pp. 4903-4909, 2017.

[13] J. Y. C. Chan, N. C. F. Tse, and L. L. Lai, "A coreless electric current sensor with circular conductor positioning calibration," IEEE Sensors Journal, vol. 62, no. 11, pp. 2922-2928, 2013.

[14] D. Wang, C. Nordman, J. M. Daughton, Z. Qian, and J. Fink, "70\% TMR at room temperature for SDT sandwich junctions with $\mathrm{CoFeB}$ as free and reference layers," IEEE Transactions on Magnetics, vol. 40, no. 4, pp. 2269-2271, 2004.

[15] G. Feng, S. Van Dijken, J. F. Feng, J. M. Coey, T. Leo, and D. J. Smith, "Annealing of $\mathrm{CoFeB} / \mathrm{MgO}$ based single and double barrier magnetic tunnel junctions: tunnel magnetoresistance, bias dependence, and output voltage," Journal of Applied Physics, vol. 105, no. 3, 2009. 